\title{
Pharmacovigilance in Pediatric Age: The Role of Family Pediatricians- Medicines for Children Research Network (FP-MCRN)
}

\author{
Ettore Napoleone ${ }^{1^{*}}$ and Cristiana Scasserra ${ }^{2}$ \\ ${ }^{1}$ President of FP-MCRN (Family Paediatricians -Medicines for Children Research Network) Onlus, Italy
}

2Legal Office of FP-MCRN (Family Paediatricians -Medicines for Children Research Network) Onlus, Italy

"Corresponding author: Ettore Napoleone, President of FP-MCRN (Family Paediatrician- Medicines for Children Research Network) Onlus, Italy, Tel: 0039 874 699298; E-mail: ettorenapoleone@tiscali.it

Received date: February 26, 2015; Accepted date: July 09, 2015; Published date: July 14, 2015

Copyright: @ 2015 Napoleone E, et al. This is an open-access article distributed under the terms of the Creative Commons Attribution License, which permits unrestricted use, distribution, and reproduction in any medium, provided the original author and source are credited.

\begin{abstract}
The conduct of pharmacovigilance for paediatric use medicines requires special attention. Childhood diseases and disorders may be qualitatively and quantitatively different from their adult equivalents. Lack of clinical studies in the paediatric population leads as a consequence to drug prescriptions on an unlicensed or 'off-label' basis, because they have not been adequately tested and/or formulated and authorised for use in appropriate paediatric age groups.
\end{abstract}

Spontaneously reported suspected adverse drug reactions remain the most important source in order to promote reasonable warning signals. Pre-marketing trials are able to provide information about the benefits of drugs but do not manage to establish a safety profile. Post Authorization Safety Studies (PASS), give much more reliable estimates of the risk of ADRs than those resulting from spontaneous reports.

An improvement Family Paediatricians (FP) Pharmacovigilance training and continuous family information on the correct use of paediatric drugs may generate a better compliance of ADRs understanding.

In this context, Family Paediatricians -Medicines for Children Research Network (FP-MCRN), ENPREMA and ENCEPP member, established with the aim of developing competence, infrastructure and education for paediatric clinical trials, plays a crucial role in paediatric Pharmacovigilance (PV), through an improvement of PV paediatricians training, a correct research methodology and very strong relationship with the families.

\section{Introduction}

The limited availability of medicines specifically designed for the paediatric age is a reality with which many generations of paediatricians have learned to deal with. Less than $15 \%$ of all drugs currently marketed and less than half of those specifically intended for children are operated on the basis of clinical trials which demonstrate specific features of risk-benefit balance in children [1-4].

Many medicines are prescribed to the paediatric population on an unlicensed or 'off-label' basis, because they have not been adequately tested and/or formulated and authorised for use in appropriate paediatric age groups. Paediatricians should be aware that the use of off label drugs increases the risk of adverse reactions and they should also try to pay much more attention when they administer drug therapies to their patients [1-5].

Regulatory authorities also need to remind health professionals about the importance of their contribution towards the process of paediatric pharmacovigilance through their reporting of adverse drug reactions (ADRs) [6]. The conduct of pharmacovigilance for paediatric use medicines requires special attention. Childhood diseases and disorders may be qualitatively and quantitatively different from their adult equivalents. The lack of reliable data in the paediatric population is associated with specific problems including: limited available safety data due to the lack of clinical trials in the paediatric population; under- or over-dosing in some age groups due to lack of pharmacokinetics data or dose-finding studies; maturation, growth and development of the paediatric population susceptible to druginduced growth and developmental disorders, as well as to delayed ADRs not findable in adults [1-5].

Pre-marketing trials are able to provide information about the benefits of drugs but do not manage to establish a safety profile [1-9]. Epidemiological post-marketing studies, which have as a strong characteristic to be directed towards the recovery of all events that occur during monitoring, give much more reliable estimates of the risk of ADRs than those resulting from spontaneous reports [1-4].

\section{The FP-MCRN (Family Paediatricians - Medicines for Children Research Network)}

The Family Paediatricians - MCRN (FP-MCRN) is an ONLUS (Noprofit-association) and it is authorised to perform the contract under the Italian law. To perform clinical trials the Family Paediatricians have to respect the Italian MD 139/2001.

Family Paediatricians (FP) FP interested in drug research and investigation must attend voluntarily specific training courses which are organized in all Italian regions and/or districts (in order to become FP- Investigators). Each FP-Investigator has his/her own database which is kept updated with information regarding his patients (about 900 children) who have been in his/her care from birth to 14 years of 
age. The data that he /she have are numerous and can be very useful for any observational study and can also be used for follow-up studies.

Furthermore it can be said that for each research study a CRF-e is carried out by software engineers, transparent, valid and consistent data can be obtained from them, their traceability can then be followed up.

The FP-MCRN therefore has access to numerous data (all FP can take care of and pursue in the follow up of more than 7 million children aged from 0 to 14 years) especially data for authorized products and data on vaccines (nationally and centrally authorised products and vaccines ). In fact, every child is pursued for the administration of vaccines and to highlight any ADRs linked to them, vaccination record cards are inserted in the database of the FP. The FP has the role of Paediatricians Sentinel in collaboration with the National Institute of Health for the management of vaccines and any ADRs after their administration.

The possibility of a long-term follow-up, and the experience gained in the conduct of clinical studies (GCP) can facilitate the realization of PASS with the ethicality, quality and scientific quality.

Through training (TRAINING AND EDUCATIONAL COURSES) specific (MD 139/2001) FP-researchers acquire in fact a correct research methodology according to GCP standards, the Ethical Consideration and the Code of Conduct of ENCePP for the conduct of clinical studies on children.

In addition, the MD 139/2001 foresees that each FP-Investigator is a center recruiter who must submit the research project to the Ethic Committee (EC) of reference for which all studies of FP-MCRN are considered multicentric.

The FP-MCRN can survey more than 7.000.000 Italian children. The merit of FP is their close elective involvement with children and their families. In Italy, a FP generally establishes a very strong and close "paediatrician-parents" and "paediatrician-child" relationship. For this reason it become easier the capacity to assess and involve outpatients in clinical trials. Practical reasons: 1) Numbers of patients 2) FP as a member of the family; 3) Direct relationship with the children; 4) Presence of territorial pathologies; 5) Frequency of the contacts out-patient both in ambulatory and domiciliary [10-12].

\section{Research Team \& Tasks}

"One of the targets of Family Paediatrics is to carry out clinical research and investigations on paediatric medicines with the ethicality and quality needed to guarantee the broadening of knowledge and the improvement of prescription appropriateness. Authorised drugs should be used only after having the results of specific studies which evaluate the effectiveness/safety profile and dosage for paediatric age groups; pharmacology culture of paediatric age groups needs to be expanded together with the necessity of offering protection to those children who participate to clinical trials, avoiding non- necessary studies, succeeding in gathering evidence about adverse drug reactions (ADRs) and establishing correct dosage for each age group. Research could and must be carried out independently by Family Paediatricians and some examples have been set thanks to the specific competence of territorial paediatrics in several areas of excellence. In some cases a "solid strategic alliance" with other characters that play a key role in the paediatric research (Universities, Hospitals, Scientific Institutes for Quality of Healthcare, Scientific Associations) should be considered and main importance should be given to a close co-operation and synergy between the areas of excellence of national paediatrics" [2].

The Family Paediatricians -Medicines for Children Research Network (FP-MCRN), ENCePP member, established with the aim of developing competence, infrastructure, networking and education for paediatric clinical trials, became a member of the ENPR-EMA responding to all the requirements. The FP-MCRN became also a member of the Coordinating Group of ENPREMA.

\section{The Main Principles of the FP-MCRN (ENCEPP Code of Conduct)}

Scientific independence, by ensuring best practice in the relationship between investigators and study funders (as reported in Italian MD n.139/2001), including protocol agreement and publication of results; and Transparency throughout the research process and when reporting results.

The studies will perform in line with the ENCEPP Code, the Guidelines for Good Pharmacoepidemiology Practices of the International Society of Pharmacoepidemiology (ISPE GPP, Revision 2, 2007) and ENCePP Guide on Methodological Standards in Pharmacoepidemiology which provides a framework of scientific guidance towards the conception and execution of pharmacoepidemiology and pharmacovigilance studies.

This strong and reliable scientific organization is funded on Standard Operational Procedures (SOPs), Quality Control (QC and QA), transparency and traceability of clinical data, validity and safety data of the single clinical trials and GCP rules.

\section{FP-MCRN Research Team}

All phases of a research project are established and organized by the Research Team in charge to manage research projects. Ensuring that all stages of the project are carried out with the scientific quality and ethics required. It is the task of the Steering Committee for each research project to establish a specific Research Team, with its Effective Members and it's External Members who are identified as Seniors Investigators or other Experts depending on the specific nature of the project.

The FP Steering Committee must express a positive opinion for the single clinical trial before this could be submitted to the National C.E. Steering Committee formed by family paediatricians who have the best competence and experience. The Steering Committee, with the responsibility and supervision of the Head of FP- MCRN, will have the task of proposing research projects, regular reviewing, and organising training courses, epidemiological investigating, attending to international conferences, magazine publishing with IF and everything which has to do with science and that will be considered useful for family paediatricians' clinical research. Moreover, the Steering Committee will control, verify and implement all the scientific activities proposed and/or suggested by each member of the RWG. Finally, the Steering Committee will have the task to verify the methodological quality of all the research projects which will then be evaluated by Ethics Committees.

\section{In this view the FP-MCRN has got}

A legal insurance office, that guarantees the legal control and the policy privacy of the trails 
The FPs - monitor that constantly follow all the enrolling centres and the norms of GCP

\section{The FP-auditors, that affect some audits to check out the QA}

All in respect of a vulnerable population such as the paediatric one. As it regards the control of the possible ADRs in the clinical trials, of the safety and of the monitoring of the follow up, this is submitted to the Safety monitoring group.

The traceability, the transparency and the safety of the data are submitted to a computer engineer, provided of certification ISO 27001 in safety subject and computer data, that has the assignment to manage the various electronic CRFs created for every single demand The informatics engineer also deals with centralized randomizations to level and randomizations in blocks or stratifies according to the various demands.

The standardization of the procedures of quality and control guarantee the immediate usage of the inserted data; there are besides present numerous controls that are activated during the insertion of the data.

\section{FP-MCRN Regulatory Activity}

Family Paediatricians foster close collaboration with the national regulatory activities through the participation in the AIFA Paediatric Working Group (PWG) until 2012. PWG was formed with the aim to create continuity between the world of paediatrics and Regulatory activity both Italian and international level. PWG was formed, with the collaboration of many experts, with a specified aim and that is to launch initiatives and measures for more rational use of the drug in children.

FP contributed to regulatory activities with a critical revision of the benefit/risk profile of some relevant paediatric drugs (e.g. sympathomimetic nasal decongestants banned below 12aa, metocloparamide prohibited below 16aa, recommendations on tropicamide and phenylephrine, domperidone and oxatomide) $[13,14]$.

A systematic off-label use of drugs has been one of the main activities of the AIFA Paeediatric-Working Group. An updated list of off-label drugs associated with the availability of scientific evidence was put together and sent to the relevant AIFA Technical Committee of Science. It 'was drawn up an updated list of off-label drugs associated with the availability of scientific evidence.

29 off-label drugs that are necessary to treat cardiovascular pathologies in children are legitimized by the PWG-AIFA. Thus, their use is authorized and they can be supplied by the National Health Service. Such drugs belong to the following classes: antiarrthymics, calcium antagonists, hypocholesterolemics, hypotensives, peripheral vessel dilators, ACE-inhibitors, beta-blockers, diuretics and sympathicomimetics. RCTs exist for 20 of these drugs; however, for the remaining 9 consensus articles or guidelines that envisage their utilization for said pathologies in children have been produced .AIFA decided to stop the PWG activities in 2012.

\section{The role of Family Paediatricians in ADRs Reporting System}

Family Paediatricians case reports mainly involve vaccines (exavalent, Anti-pneumococcal, anti-meningococcus, Trivalent measles / mumps / rubella) as they are used in most paediatric subjects, either because they involve the duty to report any type reaction also including those which are expected not to be serious. Vaccines, followed by frequency reports of pharmaceuticals used by children: antibiotics, antipyretics, specific products for colds and some drugs belonging to the class of drugs on the gastrointestinal tract and metabolism. Most of the ADRs, observed in paediatric prospective studies, mainly effect the skin (rash, urticaria) and the gastrointestinal system (diarrhea, nausea and vomiting), but we can also observe systemic reactions and reactions connected to the central nervous system $[13,14]$.

In Italy, the activity of spontaneous reporting in children is still very low: in recent years paediatric data-reporting has stood at around 1 1.6 to 1 percent, compared with $8 \%$ of total reporting (4). Reasons may be different: 1) a non-spread of an iatrogenic disease culture over the years 2) not having understood the real benefits that the spontaneous reporting system can create for the community in terms of reducing the risks of patients and saving resources.

A major cause of underreporting by family paediatricians is determined by the behaviour of families, when facing adverse reactions due to a non-rational use of drugs (often caused by the use of "Do it yourself" drugs), as they prefer to go directly to the Emergency Department [1-5].

A high percentage of the accesses to the emergency room for adverse reactions, after an ingestion of an overdose of medication, have been registered. The main causes of overdoses are: accidental ingestion of the drug for the lack of adult control, defective or inadequate packaging (for example, the lack of safety lock systems), the error in the preparation / dilution of a more concentrated drug. These also include the ingestion not being aware of the same active ingredient in drugs sold under a different name and a different indication. One example that continues to reach the RNF reports of ADRs in children is of overdose of oxatomide. There are two different packages on the market with the same dosage form but at different concentrations $(0.25 \%$ and $2.5 \%)$, which shows an increased risk of overdose linked to an incorrect administration [1-4].

In this scenario, a pilot pharmacovigilance project, named "Monitoring of the Adverse Effects in Paediatric population (MEAP)", design to enhance high quality spontaneous reporting of ADRs in children by both hospital and family paediatricians have developed [2].

Several strategies were pursued in the project all aimed enhancing high quality spontaneous reporting of ADRs in children. One of these strategies was a specific attention to formation of involved paediatricians, which was carried out at the beginning of the study but also with a follow up in the third month. In addition a periodic training on specific issues of drug safety emerging from the spontaneous reporting system was systematically performed. In addition, an electronic monthly report of the data with specific commentaries on relevant issues emerging from the study was generated and delivered to the involved paediatricians. An important and innovative achievement of the project was the information of official Italian ADRs reporting form which rendered its filling an easy to perform task leading also to a better quality ADR reporting. Taken together these measures not only ameliorated ADR reporting and quality but also led to a progressive involvement of enrolled paediatricians into a working and efficient pharmacovigilance network. 
The MEAP project demonstrates that an approach of pharmacovigilance accompanied by continuous training, sharing of results and easy to fill reporting forms, enhances the effectiveness of generating new informative data which can be exploited for benefit/ risk analysis, improving drug prescriptions and ameliorating the current regulation, thus ultimately impacting on paediatric patient safety. Nevertheless much more efforts is needed to guarantee that children will receive the correct medications, appropriate to their clinical conditions.

To understand the reasons beyond this situation we performed a survey, the first of its kind in Italy, to evaluate FPs knowledge, feeling and compliance in ADR reporting [15]. A total of 552 FPs evenly distributed throughout the Italian territory provided a feedback to the survey. Knowledge of pharmacovigilance (PV) resulted to be poor, mainly due to the absence of adequate training in academy; despite this, the majority of FPs declared to be interested to PV and aware of its positive impact on their clinical practice. Yet, FPs reported a poor compliance to the reporting of ADRs. A very high variability in ADRs reporting however was observed among the regions, possibly because of variability of regional educational programmes dedicated to PV.

The level of FPs knowledge on PV was assessed through 11 questions focused on definition of ADR, the differences between ADR in children and adults and phytovigilance. Almost $80 \%$ of the participants were unable to answer correctly on the definition of adverse drug event (ADE) (correct answer mean 21\%; 95\% CI 12$31 \%$ ), while the majority of them responded correctly on the definition of ADR (correct answer mean 96\%, 95\% CI 94-98\%). More-over, 50\% of FPs were unable to identify the right definition of spontaneous ADR (correct answer mean 53\%, 95\% CI46-62\%), or the class of drug most frequently associated to ADRs in children (correct answer mean 59\%, 95\% CI49-69\%). FPs, however, displayed a good knowledge of PV.

Four items dealt with PV perception, asking also to evaluate specific interventions aimed at improving the ADR's report rate. Most of FPs considered the academic preparation in PV "insufficient" (76.5\%) and only $1 \%$ of the responders defined their preparation as "exhaustive". However, 92\% of FPs had the perception that PV can improve their patients care and $78 \%$ would be willing to participate in specific training, if made available.

This study provides the first Italian survey on paediatric PV. It highlights that FPs are aware of PV importance in their clinical practice while recognising an insufficient education on it. Considering these observations, we believe that an improvement of PV academic training, continuous training and information at the territorial level, possibly coordinated at the national and transnational levels may generate a better compliance and thus optimisation of ADRs reporting".

\section{FP-MCRN Training and Educational}

There is the need to awaken FPs to a culture of iatrogenic disease, to the reporting of ADRs and to the proper use of reporting forms. On the other hand there is a need for a greater culture of research in paediatric pharmacology leading towards an increasingly pro-active PV.

We organized three Pharmacovigilance Courses directed to Family Paediatricians on these subjects. The construction of specific expertise in research methodology was to: 1) constitute that expertise paediatric that are requested of research quality; 2) increase, implement, and extend the most possible the national network of excellence FP-MCRN already existing on the national territory of Paediatricians Investigator. This through two levels of training: 1) a first level, with the formation of a significant number of paediatricians investigators (DL 139/2001) in order to promote the qualitative and quantitative growth of the network 2) a second level, with the formation of a pool of paediatricians investigators with skills higher to ensure a further leap of quality of the studies of bugs.

We organized also two Family paediatricians Research School in 2011 and in 2013: FP-Investigators acquired in fact a correct research methodology according to GCP standards, the Ethical Consideration and the Code of Conduct of ENCePP for the conduct of clinical studies on children and a good knowledge of Paediatric Pharmacovigilance.

Through training courses specific to the FP-Investigators have been given all the means and the rigor required for the acquisition of methodology to avoid conflicts of interest, as reiterated in MD 139/2001. It will also be of concern of the FP-MCRN -ONLUS from this moment on to sign a declaration regarding no conflicts of interest to all its members and all FP Investigators that participate in clinical tests.

\section{Clinical Research Projects}

\section{Post Authorisation Safety Studies - PASS}

When the drug is used in clinical practice in large unselected populations, PASS are useful as they find their major confirmation in recalling all the events that occur during monitoring, with estimates of incidence of ADRs that cannot be obtained by spontaneous reports. In these studies a significant role can be played by the FP with the participation to active pharmacovigilance projects.

In the study: Antibiotics Tolerability Profile Study in children with pharyngotonsillitis and rhinosinusitis [16], the FP-MCRN wanted to promote a study of the assessment of tolerability in the days of administration of Cefaclor compared with amoxicillin-clavulanate. The primary objective of the Study was to assess in patients with pharyngotonsillitis (FT) and rhinosinusitis (RS) the tolerability profile of Cefaclor and Amoxicillin + Clavulanic acid after the days of administration. For the group of patients assigned to treatment arm the regime of Cefaclor dosage was $50 \mathrm{mg} / \mathrm{kg} /$ day for 5 days for the FT and for 14 days for the RS, for the one with amoxicillin + Ac. Clavulanic it was $50 \mathrm{mg} / \mathrm{kg} /$ day for 10 days for the FT and for 14 days for R.S. A total of 537 patients of both sexes, between 4 and 14 years of age were enrolled, including 435 diagnosed with pharyngotonsillitis (FT) and 102 cases with a rhinosinusitis (RS) diagnosis.

No major reactions were reported. Reported minor adverse reactions were: nausea, vomiting, diarrhea, abdominal pain, rashes, itching, loss of appetite, bloating, borborygmi, constipation, change in the hive, irritability.

In the Cefaclor-group (257 children): 26 children (10,1\%) have reported only one minor reaction, $41(16 \%)$ more than one minor reaction and $190(73,9 \%)$ did not report any reactions.

In the Amoxicillin-Clavulanic Acid group (262 children), 37 children $(14,1 \%)$ have reported only one minor reaction and 71 $(27,1 \%)$ more than one minor reaction, while 154 (58,8\%) did not report any reactions. Minor adverse reactions, mainly gastrointestinal 
Page 5 of 6

plead for a better Cefaclor tolerability $(\mathrm{p}<0.001)$ when comparing Cefaclor to Amoxi-clavulanate.

\section{AIFA Pharmacovigilance 2008-2009 -Call}

\section{Active surveillance on the use of antibiotics in children, especially in the age group between 0 and 2 years"}

The Italian Medicines Agency (AIFA) approved, for the first time ever, a project conceived, structured and implemented by the Family Paediatrician and presented by the Molise Region, within the program of active pharmacovigilance funded through the regional funds available for the years 2008 and 2009.: Active surveillance on the use of antibiotics in children, especially in the age group between 0 and 2 years."

"From the data OSMED of prescription of pharmaceuticals in which a widespread of antibiotics were calculated, stratified by age, once again there emerges an" over-prescription "of these drugs, especially in the age group between 0 and 2 years an increase in adverse reactions related to them is noted.

It still seems obvious that inefficient use of these drugs as instructed are not always based on scientific evidence raising the problem of antibiotics- strength and quality of the therapy.

Hence the need for a research project, Phase IV, managed and coordinated in full for the first time with the Family Paediatrician, that can foresee on one hand training and information regarding the proper use of antibiotics in children and possible iatrogenic pathologies caused by their improper use, the other to constitute a territorial survey in the prescriptive appropriateness and safety of these drugs in children aimed at evaluating the risk-benefit balance on usage.

The study wants to firstly exceed the limitations of the data OSMED they see as an impossibility to track the diagnosis that is the basis of the prescription, and of a correct valuation of prescriptions in the various paediatric age groups (especially $0-2$ years).

Considering the national and international guidelines (NICE 2008 etc.) on the correct use of antibiotics in pediatrics, the possibility of an enrollment of about $100 \%$ of patients, instrumental examinations at their clinics (self-help) collaboration with families and proper adherence to the protocol, the study conducted by the FP will allow a general improvement of the appropriateness of prescriptions, having a clear picture of the epidemiological situations gaining effective control of the benefits of the therapy, a reduction in the number of relapses (resistance antibiotics) and a proper evaluation of those that are the possible ADRs related to the therapy.

Leastways, the study will encourage savings in pharmaceutical expenditure taken on by the SSN together with a reduction of prescriptions through a correct diagnosis but mainly a reduction of inappropriate hospital admissions. "

The project will have a lifespan of about one year and the objectives are the improvement of the appropriateness of prescriptions and of the proper diagnosis, reducing inappropriate hospital admissions in paediatrics and achieving the reduction of the consumption of pharmaceutical expenditure relating to the use of antibiotics in the age group of $0-2$ years.

\section{AIFA Pharmacovigilance 2010-2011 -Call: REDS (Respiratory Drugs Survey) Study}

\section{Active surveillance of respiratory drugs, especially inhaled steroids (CSI) in children}

Despite the growing campaigns undertaken by the AIFA, the consumption and unsuitable prescription for certain categories of medicines, above all antibiotics and respiratory medications, especially inhaled corticosteroids (CSI) in the age group 0 to 14 years with an increase adverse reactions related to them.

In particular, the data OSMED 2013 reported that $28 \%$ of children aged 0 to 4 years received at least one drug regarding the respiratory system with the increase in consumption of ' $1.1 \%$ over the previous year, making it evident of non-rational use of these drugs which are not always based on scientific evidence.

Guidelines of reference for the treatment of asthma (GINA- Global Initiative for Asthma) have been well-established for years, and, despite the prevalence of this disease established between 9.5 and $10.5 \%$ in children aged 6 to 11 years, it is observed as the asthma medicines in particular CSI (Beclomethasone, budesonide, flunisolide, fluticasone, etc.), are used inappropriately in pediatrics with a "overprescription" for conditions that do not require the use.

For example, Beclomethasone is one of the most frequently prescribed drugs in children. It is estimated that it is consumed in Italy every year by about 2 million children and adolescents. Despite being an anti-asthmatic drug is often prescribed for colds, coughs and sore throats, so that Italian children have a 3 times higher probability to receive an anti-asthmatic drug rather than their peers living in other European countries.

An independent clinical trial was conducted to evaluate the efficacy of Beclomethasone in the prophylaxis of bronchospasm due to viral infections of the airways (the so-called viral wheezing). In the ENBe study (Effectiveness of Beclomethasone versus placebo in the prophylaxis of viral wheezing in preschool) Beclomethasone reduced (from 11 to $7 \%$ ) the risk of viral wheezing by $4 \%$ but the difference was not statistically significant [17].

The results of Enbe Study confirm a fact already highlighted by some studies: the CIS have a modest efficacy in preventing both recurrent wheezing viral and infections by respiratory syncytial virus (bronchiolitis) in the child. In addition, no benefits were seen in reducing symptoms of respiratory tract infection. Therefore, a more rational use of these drugs is necessary, both by doctors but also by parents who frequently administer the drug to the child with infections of the respiratory tract, without consulting their paediatrician.

From these considerations comes the need for a research project Phase IV conducted in the territory led by Family paediatricians that can on the one hand predict the FP training courses and information on families regarding the use of respiratory medication the CIS and any iatrogenic condition caused by their improper use, on the other hand which may constitute an active territorial survey on prescriptive appropriateness and safety of these drugs in children aimed at evaluating the risk-benefit balance on usage.

The study wants to first of all overcome the limitations of the OSMED data seen as the inability to track the diagnosis behind the prescription and of a proper valuation of prescriptions in the various 
paediatric age groups. This study will enable a wide spread to the FP Guidelines Reference and implement skills (Training FP) for a correct diagnosis, to a viable therapy and close follow up of pathologies that require the use of these drugs in all children from 0 to 14 years regarding their clinical seriousness.

Finally, the study will encourage closer synergy between FP and families through better and more accurate information given to families on the use of respiratory drugs and in particular of CIS [Pharmacoepidemiol. Drug Sat. 2014.23 [4], 406-10] will lead to a reduction of the "do it yourself" and self-prescription for illnesses that do not include their use in LG and will certainly lead to a reduction on working days lost by parents and of any hospitalizations (reducing inappropriate admissions).

All this together with an information campaign will include, direct contact with the families of the FP through specific information talks, the creation of posters, brochures and possibly CDs direct to parents and on display in FP offices (and / or delivered directly to the families) and / or in the media (newspapers and TV) on the correct use of drugs in paediatric respiratory (and in particular the CIS). All verifiable later with the satisfaction questionnaires given to parents.

\section{Conclusion}

A future path to follow in paediatrics is undoubtedly towards the synergy and coordination of all components of Paediatrics (universities, hospitals, territories) for the organization of research projects and post-marketing programs. The starting point must necessarily be cultural: an implementation of the culture of iatrogenic disease and a careful assessment of the significance of PAS Studies by Family Paediatricians.

There is the need to awaken PDFs to a culture of iatrogenic disease, to the reporting of ADRs and to the proper use of reporting forms. On the other hand there is a need for a greater culture of research in paediatric pharmacology leading towards an increasingly pro-active FVG.

The organization of specific PV training courses and research projects should meet the following objectives: 1) promote the culture of iatrogenic disease in paediatrics providing information on the benefit-risk profile of drugs. 2) Encourage spontaneous reporting of ADRs in children in frequency and quality both in hospital and family paediatricians ambulatory.

Of course, all these supported by capacity building of the Network of FP-Investigators which is central to PASS and DBPC-RCT studies.

The creation of a specific database managed and coordinated by the FP must also results becomes strategic and very important for a careful and correct evaluation of ADRs. Last but not least is the fact that with the FP there would be fewer drop-outs in LTFU if there is a taking charge of children and establishing a close relationship with families.

Quality and ethicality of research are becoming important endpoints of FP-MCRN, opportunities and challenges of Family
Paediatricians during their education and during the sponsoring of health and prevention in the paediatric age groups.

\section{References:}

1. Napoleone E (2010) Children and ADRs (Adverse Drug Reactions). Ital J Pediatr 36: 4 .

2. Napoleone E, Radice S (2012) From pharmacovigilance to therapy amelioration in paediatric patients: The role of the clinical pharmacologists and family paediatricians Part of a series on Paediatric Pharmacology, guest edited by Gianvincenzo Zuccotti, Emilio Clementi, and Massimo Molteni. Pharmacol Res 65:168-170.

3. Napoleone E (2009) Medicines for children ADRs: from spontaneous reporting to active souveillance. Child :care, health and development 36: 106-107.

4. Filograna MR, Clementi, Napoleone E, Radice S (2011) La Progettualità in farmacovigilanza: dal reporting alla safety in età pediatrica. ILMEDICOPEDIATRA 2: 20 -27.

5. D Amore C, Menniti-Ippolto F, Traversa G (2014) La Farmacovigilanza in età pediatrica. Prospettive in Pediatria 44: 203-207.

6. Regulation (EC) No $1901 \mathrm{f} 2006$ of Ihc European Parliament and of the Council of 12 December 2006 on medicinal products for paediatric use.

7. Blake KV, Zaccaria C, Domergue F, La Mache E, Saint-Raymond A, et al. (2014) Comparison between paediatric and adult suspected adverse drug reactions reported to the European medicines agency: implications for pharmacovigilance. Paediatr Drugs 16: 309-319.

8. Palmaro A, Bissuel R, Renaud N, Durrieu G, Escourrou B, et al. (2015) Off-label prescribing in pediatric outpatients. Pediatrics 135: 49-58.

9. Aagaard L, Hansen EH (2014) Adverse drug reactions in children reported by European consumers from 2007 to 2011. Int J Clin Pharm 36: 295-302.

10. Napoleone E. (2011) The FIMP-MCRN (Family Paediatricians Medicines for Children Research Network): generating evidence and safety at the point of care. Evid.-Based Child Health 6: 37.

11. Napoleone E (2011) Excellence in Family Paediatricians: the FIMPMCRN (Medicines for Children Research Network) becomes a member of ENPR-EMA (European Network of Paediatric Research at the European Medicines Agency). Ital J Pediatr 37: 7.

12. Napoleone E, Mele G (2010) Medicines for children: the proactive approach of the FIMP-MCRN Family Paediatricians - Medicines for Children Research Network. ACTA PEDIATRICA 99: 462-113.

13. Segnalazioni spontanee di sospette reazioni avverse in età pediatrica nel 2008 (2009) BIF - AIFA.XVI 2: 79-82.

14. Ferrajolo C, Capuano A, Trifirò G, Moretti U, Rossi F, et al. (2014) Pediatric drug safety surveillance in Italian pharmacovigilance network: an overview of adverse drug reactions in the years 2001 - 2012. Expert Opin Drug Saf 13 Suppl 1: S9-20.

15. Pellegrino P, Carnovale C, Cattaneo D, Perrone V, Antoniazzi S, et al (2013) Pharmacovigilance knowledge in family paediatricians. A survey study in Italy. Health Policy 113: 216-220.

16. Bottaro G, Biasci P, Lo Giudice M, Mele G, Montanari G, et al. (2012) [5 days Cefaclor vs. 10 days amoxicillin/clavulanate in the treatment of childhood streptococcal pharyngitis. Data from a randomized clinical trial]. Minerva Pediatr 64: 341-346.

17. Clavenna A, Sequi M, Cartabia M, Fortinguerra F, Borghi M, et al. (2014) Effectiveness of nebulized beclomethasone in preventing viral wheezing: an RCT. Pediatrics 133: e505-512. 